\title{
Reliability modelling for the GIL subject to competing failure modes
}

\author{
Songhua Hao ${ }^{1}$, Jun Yang ${ }^{1,{ }^{*}}$, Bo Cheng ${ }^{2}$, Yun Chen ${ }^{3}$ and Boyuan Cui ${ }^{3}$ \\ ${ }^{1}$ School of Reliability and Systems Engineering, Beihang University, Beijing, 100191, China \\ ${ }^{2}$ Nuclear Power Institute of China, Chengdu, 610213, China \\ ${ }^{3}$ China Electric Power Research Institute, Beijing, 100085, China
}

\begin{abstract}
Due to necessity of enforcing the electrical transmission network, a long distance gas insulated transmission line (GIL) has been developed as a new alternative underground technology, and has shown an ever-increasing usage worldwide. The main failure modes of the GIL are performance degradation due to air leakage, and random shocks caused by partial discharge. The failure of the GIL subject to both soft failure and hard failure is the result of their competition. Considering that the degradation occurs only after a certain delay, i.e. there is a random degradation initiation time before the air in the GIL begins to leak, this paper presents a new reliability model for the GIL subject to competing failure modes. Its reliability is assessed through analytical derivation and numerical calculation methods. And a numerical study is conducted to illustrate the implementation of the proposed model.
\end{abstract}

Key words. the GIL, partial discharge, air leakage, degradation initiation time, reliability.

\section{Introduction}

To meet the necessity of enforcing the electrical transmission network, and considering that erecting new overhead lines may encounter many insurmountable difficulties for some specific environment and terrain, a new alternative underground technology is developed as a long distance GIL [1]. Based on the successful SF6 tubular conductor technology, the first GIL was commissioned in 1975 at Wehr pumped storage station in the Black Forest in Southern Germany [2]. Since then, the GILs at rated voltages of $110,225,420,550$ and up to $1100 \mathrm{kv}$ have shown an ever-increasing usage worldwide, and the GIL technique has been proven to be in high reliability in terms of mechanical and electrical failures [3].

Similar to the gas insulated transmission switchgear (GIS), the failure of the GIL is mainly due to partial discharge and air leakage. Due to the existence of defects like abnormal conductor surface roughness and metallic particles, partial discharge occurs and finally leads to a break down [4]. Therefore, guidance for the design, installation, testing, operation and other technical aspects of the GIL is provided in [5], including the partial discharge tests on the insulators. And newly developed measuring system are studied in many literature such as [6]. Besides hard failure caused by partial discharge, soft failure caused by air leakage can also be found in many applications, e.g., Jinping-I Hydropower Station in China
[7] and Hainan nuclear power unit 1 in China [8]. And the main reason of SF6 leakage of the GIL is the degradation of the sealing ring [9].

Based on practical failure analysis in [7], it can be indicated that the defect of the sealing ring will not cause air leakage as soon as the GIL is put into service. In practice, sometimes the degradation occurs only after a certain delay, called the onset time or the initiation time. In [10], Peng and Feng developed a reliability model for Ultrathin Gate Oxides subject to Verhulst logistic growth model with random onset time. Based on a Wiener degradation process and exponentially distributed random initiation time, Paroissin studied the corresponding parameter estimation methods and some asymptotic results [11]. To characterize the occurrence of failure caused by partial discharge, Wang et.al presented a compound Poisson process and planned the maintenance strategy under comprehensive consideration of optimal reliability and economic efficiency [12].

Considering the competing failure modes of the GIL, i.e., hard failure due to partial discharge and soft failure caused by air leakage, this paper proposes a new reliability model with random degradation initiation time before the air in GIL begins to leak. A nonlinear general path model is developed to model the aging of the GIL sealing rings, which is assumed to be the primary cause of air leakage. Besides, a Poisson process is utilized to model the occurrence of partial discharge, which will

\footnotetext{
* Corresponding author: tomyj2001@buaa.edu.cn
} 
cause the GIL failure according to the extreme shock model [13], i.e., the voltage exceeds the partial discharge inception voltage (PDIV) of the GIL. Finally, a competing failure model is proposed to assess the reliability of GIL system.

The rest of this paper is organized as follows. Section 2 describes the competing failure modes of the GIL. System reliability model for the competing failure modes is proposed in Section 3. A numerical example is developed in Section 4 to demonstrate the implementation and effectiveness of the proposed model. And some conclusions are finally given in Section 5.

\section{Competing failure modes of the GIL}

As shown in Figure 1, the failure of the GIL is the competing result of soft and hard failures. A degradation based soft failure occurs when the air leakage, which begins after a random initiation time, exceeds a critical threshold level H. In addition, a shock based hard failure occurs once a partial discharge with large voltage arrives. The whole description of the system and relevant denotations are expounded below.

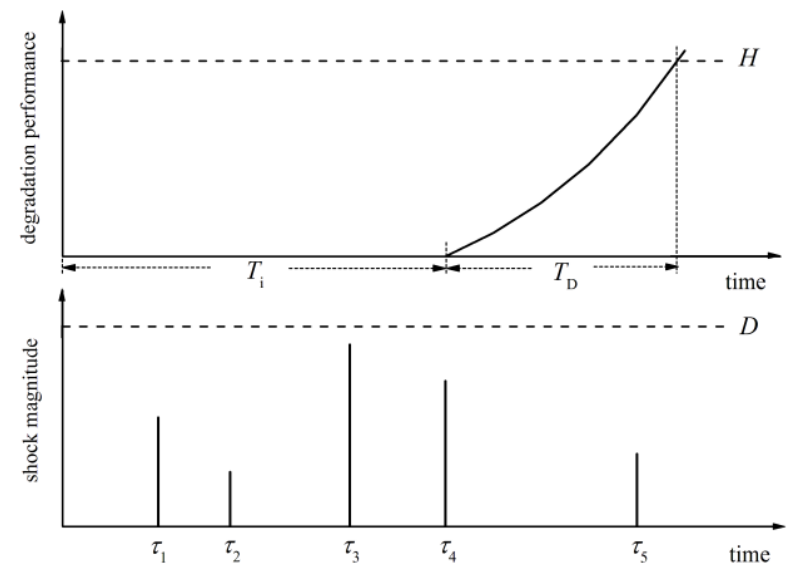

Figure 1. Competing failure modes of the GIL.

1) The degradation process $X(t)$ is modeled by a nonlinear general path model [14] with random initiation time $T_{i}$. After the degradation initiation time, i.e., in the degradation propagation stage, the degradation performance can be defined as

$$
X(t)=\lambda \Lambda(t)+\varepsilon
$$

where $\Lambda(t)$ is the time-transformed function of degradation path. $\lambda \sim N\left(\mu_{\lambda}, \sigma_{\lambda}^{2}\right)$ is the random effect parameter representing the unobservable endogenous factors of samples. $\varepsilon \sim N\left(0, \sigma_{\varepsilon}^{2}\right)$ is the model error term.

2) Similar to the assumption in literature [11] and [15], $T_{i}$ is assumed to obey an exponential distribution in this article, i.e., $T_{i} \sim E\left(\lambda_{i}\right)$. Considering that the air leakage of the GIL is mainly due to the aging of sealing rings, an exponential form is utilized to characterize it, i.e., $\Lambda(t)=e^{K t}-1$, which is common in the degradation modelling for rubber sealing rings such as [16] and [17]. Therefore the nonlinear general path model (1) can be further expressed as

$$
X(t)=\lambda\left(e^{K t}-1\right)+\varepsilon
$$

3) Shocks arrive at a homogenous Poisson process with a constant rate $\lambda_{0}$. The number of arrived shocks before time $t$ is denoted by $N(t)$, and $P\{N(t)=k\}=\frac{\left(\lambda_{0} t\right)^{k} e^{-\lambda_{0} t}}{k !}, k=0,1,2, \cdots$ The magnitude $W$ of shocks is independent and normally distributed variable $W \sim N\left(\mu_{W}, \sigma_{W}^{2}\right)$, and its cumulative distribution function (cdf) is denoted by $F_{W}(x)$;

4) The threshold levels for soft and hard failures are constants $H$ and $D$, respectively. Once the overall degradation performance exceeds $H$, or the magnitude of a shock is greater than $D$, the GIL fails.

\section{Reliability modeling for the GIL}

The failure of the GIL subject to both soft failure and hard failure is the result of their competition. Considering these two failure modes are independent with each other, this Section respectively derives the reliability functions for soft and hard failures, and the system reliability is finally obtained as the product of them.

$$
R(t)=R_{S F}(t) \cdot R_{H F}(t)
$$

Let $F_{i}(t)$ and $f_{i}(t)$ be the cdf and pdf of exponentially distributed degradation initiation time $T_{i}$, respectively. Then it can be derived that:

$$
f_{i}(t)=\lambda_{i} e^{-\lambda_{i} t}, t>0
$$

Based on the degradation model (2), and considering that $\lambda, \varepsilon$ are independent normally distributed variables, it can be derived that $X(t)$ also follows a normal distribution with mean $\mu_{\lambda}\left(e^{K t}-1\right)$ and standard deviation $\sqrt{\sigma_{\lambda}^{2}\left(e^{K t}-1\right)^{2}+\sigma_{\varepsilon}^{2}}$. Denote the degradation propagation time before failure by $T_{D}$, then its cdf $F_{D}(t)$ can be derived as 


$$
\begin{aligned}
& F_{D}(t)=P\left\{T_{D}<t\right\} \\
& =P\left\{\lambda\left(e^{K t}-1\right)+\varepsilon<H\right\} \\
& =\Phi\left\{\frac{H-\mu_{\lambda}\left(e^{K t}-1\right)}{\sqrt{\sigma_{\lambda}^{2}\left(e^{K t}-1\right)^{2}+\sigma_{\varepsilon}^{2}}}\right\}
\end{aligned}
$$

As shown above in Figure 1, the soft failure life $T$ is determined by the overall degradation process, which consists of degradation initiation time $T_{i}$ and degradation propagation time $T_{D}$. Therefore, the pdf of $T$ can be obtained by using a convolution integral of $T_{i}, T_{D}$.

$$
\begin{aligned}
& R_{S F}(t)=P\{T>t\}=P\left\{T_{i}+T_{D}>t\right\} \\
& =\int_{0}^{+\infty} P\left\{v+T_{D}>t\right\} f_{i}(v) d v \\
& =\int_{0}^{+\infty} P\left\{T_{D}>t-v\right\} f_{i}(v) d v \\
& =\int_{0}^{+\infty} F_{D}(t-v) f_{i}(v) d v \\
& \left.=\int_{0}^{+\infty} \Phi\left\{\frac{H-\mu_{\lambda}\left[e^{K(t-v)}-1\right]}{\sqrt{\sigma_{\lambda}^{2}\left[e^{K(t-v)}-1\right]^{2}+\sigma_{\varepsilon}^{2}}}\right\} d F_{i}(v)\right]
\end{aligned}
$$

The complexity of the integration function forms a challenge on the computation of Equation (6). Considering the fact that $v \in(-\infty,+\infty)$ corresponds to $F_{i}(v) \in[0,1]$, a numerical integration method can be adopted to calculate Equation (6) as follows:

$$
\begin{aligned}
R_{S F}(t) & \left.=\int_{0}^{+\infty} \Phi\left\{\frac{H-\mu_{\lambda}\left[e^{K(t-v)}-1\right]}{\sqrt{\sigma_{\lambda}^{2}\left[e^{K(t-v)}-1\right]^{2}+\sigma_{\varepsilon}^{2}}}\right\} d F_{i}(v)\right] \\
& =\int_{0}^{1} \Phi\left\{\frac{H-\mu_{\lambda}\left[e^{K\left(t-F_{i}^{-1}(u)\right)}-1\right]}{\sqrt{\sigma_{\lambda}^{2}\left[e^{K\left(t-F_{i}^{-1}(u)\right)}-1\right]^{2}+\sigma_{\varepsilon}^{2}}}\right\} d u \\
& \cong \frac{1}{N_{0}} \sum_{j=1}^{N_{0}} \Phi\left\{\frac{H-\mu_{\lambda}\left[e^{K\left(t-F_{i}^{-1}\left(\frac{j}{N_{0}}\right)\right.}-1\right]}{\sqrt{\sigma_{\lambda}^{2}\left[e^{K\left(t-F_{i}^{-1}\left(\frac{j}{N_{0}}\right)\right)}-1\right]}+\sigma_{\varepsilon}^{2}}\right\}
\end{aligned}
$$

Considering that the reliability for hard failure varies when different numbers of shocks arrive, the probability of surviving from soft failure can be derived on condition of a fixed $N(t)$.

$$
\begin{aligned}
R_{H F}(t) & =\sum_{k=0}^{+\infty} R_{H F}(t \mid N(t)=k) \cdot P(N(t)=k) \\
& =\sum_{k=0}^{+\infty}\left[F_{W}(D)\right]^{k} \cdot \frac{\left(\lambda_{0} t\right)^{k} e^{-\lambda_{0} t}}{k !} \\
& =\sum_{k=0}^{+\infty}\left[\Phi\left(\frac{D-\mu_{W}}{\sigma_{W}}\right)\right]^{k} \cdot \frac{\left(\lambda_{0} t\right)^{k} e^{-\lambda_{0} t}}{k !}
\end{aligned}
$$

Therefore, the system reliability function can be derived as.

$$
\begin{aligned}
R(t) & =R_{H F}(t) \cdot R_{S F}(t) \\
& \cong \frac{1}{N_{0}} \sum_{j=1}^{N_{0}} \Phi\left\{\frac{H-\mu_{\lambda}\left[e^{K\left(t-F_{i}^{-1}\left(\frac{j}{N_{0}}\right)\right)}-1\right]}{\sqrt{\sigma_{\lambda}^{2}\left[e^{K\left(t-F_{i}^{-1}\left(\frac{j}{N_{0}}\right)\right)}-1\right]^{2}+\sigma_{\varepsilon}^{2}}}\right\} \\
& \times \sum_{k=0}^{+\infty}\left[\Phi \left(\frac{\left.\left.D-\mu_{W}\right)\right]^{k} \cdot \frac{\left(\lambda_{0} t\right)^{k} e^{-\lambda_{0} t}}{k !}}{\left.\left.\sigma_{W}\right)\right]^{k}}\right.\right.
\end{aligned}
$$

\section{Numerical example}

To illustrate the implementation of the proposed reliability model, a realistic example is studied in this Section. Based on some reasonable assumptions for illustration, Table 1 lists the relevant model parameter values. And according to Equation (9), the reliability assessment results of the GIL, from its installation time up to 20 years, are calculated and presented in Figure 2. It is shown that the reliability of the GIL remains at a relatively high level at first for about 5 years, which is mainly due to the degradation initiation time, whose mean is identical to 5 too. After that, the reliability curve presents a sharp decline until the $20^{\text {th }}$ year, when the sealing rings of the GIL degrade greatly and the SF6 in the GIL are insufficient to maintain a good arcquenching property.

Table 1. Model parameter values.

\begin{tabular}{|c|c|c|c|}
\hline Parameter & Value & Parameter & Value \\
\hline$\mu_{\lambda}$ & 2 & $H$ & 2.5 \\
\hline$\sigma_{\lambda}$ & 0.3 & $\lambda_{0}$ & 3 \\
\hline$K$ & 0.2 & $\mu_{W}$ & 1.2 \\
\hline$\sigma_{\varepsilon}$ & 0.2 & $\sigma_{W}$ & 0.1 \\
\hline$\lambda_{i}$ & 5 & $D$ & 1.5 \\
\hline
\end{tabular}




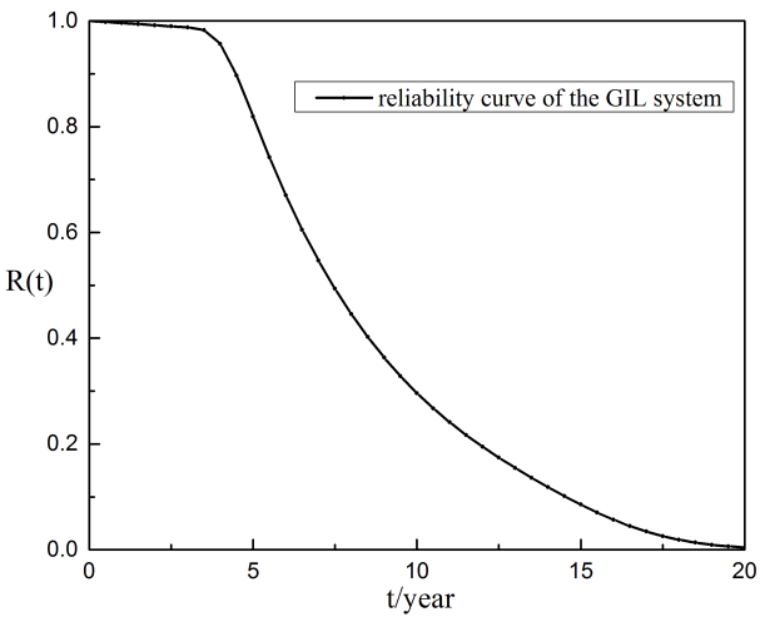

Figure 2. Reliability curve of the GIL.

Furthermore, considering $\lambda_{i}$ and $\lambda_{0}$ are two critical parameters involving the design and manufacturing process, sensitivity analysis is performed to measure the effect of them on the reliability, and the analysis results are plotted in Figures 3 and 4. It can be indicated that the reliability assessment results show remarkable sensitivity on $\lambda_{i}$, and the effect of $\lambda_{0}$ cannot be negligible, either. A larger $\lambda_{i}$ leads to a longer degradation initiation time and thus resulting in higher system reliability. And as $\lambda_{0}$ increases, the frequency of random shocks enlarges and the probability of hard failure increases, which makes the reliability of the GIL decreases.

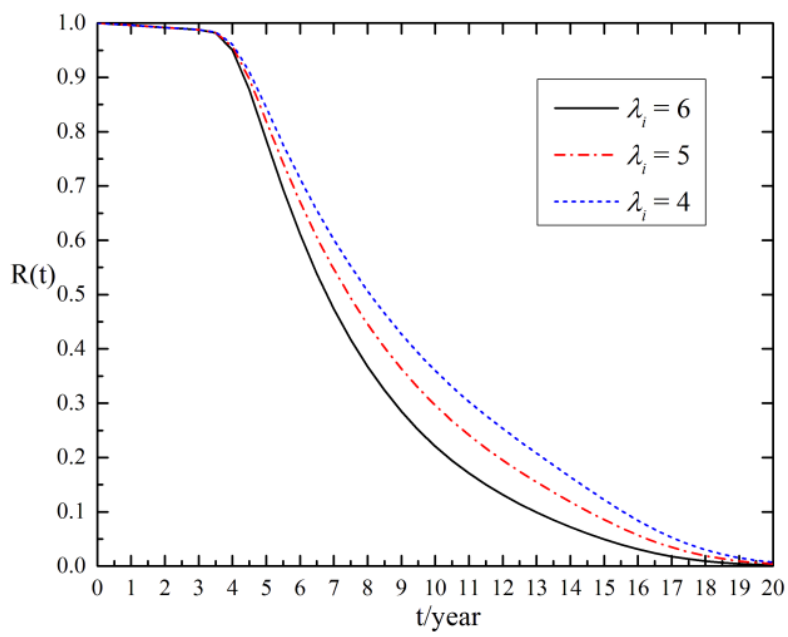

Figure 3. Sensitivity analysis of $\lambda_{i}$.

Guided by the sensitivity analysis results, in order to obtain larger system reliability, efforts should be made to increase the value of $\lambda_{i}$ and decrease the value of $\lambda_{0}$. Some suggestions can be: 1) strengthening the quality control and management of the manufacturing process, which can reduce the quantity of potential defects in the GIL and prolong the degradation initiation time; 2) improving system design and take steps to reduce the occurrence probability of partial discharge, e.g., setting a particle catcher to collect charged particles in the GIL.

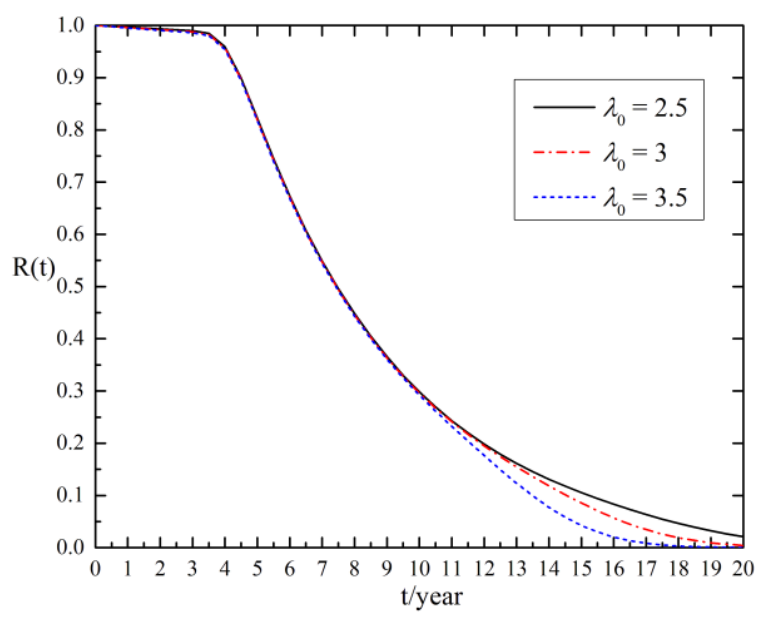

Figure 4. Sensitivity analysis of $\lambda_{0}$.

\section{Conclusions}

The primary competing failure modes of the GIL are soft failure due to air leakage, and hard failure caused by partial discharge. Taking into account random degradation initiation time before the air in the GIL begins to leak, this paper proposes a new competing failure model, and derives the reliability assessment results through analytical derivation and numerical calculation methods. Furthermore, a realistic example is studied to illustrate the implementation of the proposed model. Sensitivity analysis is conducted to measure the effect of $\lambda_{i}$ and $\lambda_{0}$ on the reliability assessment results. It is indicated that a larger $\lambda_{i}$ or a smaller $\lambda_{0}$ will lead to higher system reliability. For obtaining larger reliability of the GIL, it is suggested to make effort to increase the value of $\lambda_{i}$ and decrease the value of $\lambda_{0}$, e.g., strengthening the quality management of the manufacturing process and reducing the quantity of potential defects, or improving the system design by setting a particle catcher to collect charged particles.

\section{Acknowledgement}

This work was supported partially by National Natural Science Foundation of China (71672006), Science and Technology Project of State Grid Corporation of China (SGTYHT/15-JS-191) with title "Reliability analysis of long-distance ultra-high voltage GIL equipment".

\section{References}

1. R. Benato and D. Napolitano, 'Reliability Assessment of EHV Gas-Insulated Transmission 
Lines: Effect of Redundancies', IEEE Trans. Power Deliv., vol. 23, no. 4, pp. 2174-2181, Oct. 2008.

2. G. P. Baer, A. Diessner, and G. F. Luxa, ' 420 kV SF6-insulated tubular bus for the Wehr pumpedstorage plant electric tests', IEEE Trans. Power Appar. Syst., vol. 95, no. 2, pp. 469-477, Mar. 1976.

3. H. Koch and A. Schuette, 'Gas insulated transmission lines for high power transmission over long distances', Electr. Power Syst. Res., vol. 44, no. 1, pp. 69-74, Jan. 1998.

4. H. Okubo, M. Yoshida, T. Takahashi, T. Hoshino, M. Hikita, and A. Miyazaki, 'Partial discharge measurement in a long distance SF6 gas insulated transmission line (GIL)', IEEE Trans. Power Deliv., vol. 13, no. 3, pp. 683-690, Jul. 1998.

5. 'IEEE Guide for Application and User Guide for Gas-Insulated Transmission Lines, Rated $72.5 \mathrm{kV}$ and Above', IEEE Std C371224-2016, pp. 1-49, May 2017.

6. A. Miyazaki et al., 'Results of Partial Discharge Measurements in a Long-Distance 275 kV GIL', IEEE Power Eng. Rev., vol. 22, no. 10, pp. 58-58, Oct. 2002.

7. Y. Li et.al, 'Two cases of failure reason and treatment analysis for gas insulated transmission line (GIL)', Electronic age, vol. 0, no. 11, pp. $66-67$, 2016. (In Chinese)

8. C. Pan, 'The analysis on the reason of the GIL air leakage in 220kV system', Guizhou Electronic Power technology, vol. 17, no. 6, pp. 71 - 73, 2014. (In Chinese)

9. R. Huang et al., 'Study on aging of material for GIS sealing ring', in TENCON 2015 - 2015 IEEE Region 10 Conference, 2015, pp. 1-4.

10. H. Peng and Q. Feng, 'Reliability Modeling for Ultrathin Gate Oxides Subject to Logistic Degradation Processes with Random Onset Time: Reliability Modeling for Ultrathin Gate Oxides', Qual. Reliab. Eng. Int., vol. 29, no. 5, pp. 709-718, Jul. 2013.

11. C. Paroissin, 'Inference for the Wiener Process With Random Initiation Time', IEEE Trans. Reliab., vol. 65, no. 1, pp. 147-157, Mar. 2016.

12. Q. Wang, Z. He, S. Lin, and Z. Li, 'Failure Modeling and Maintenance Decision for GIS Equipment Subject to Degradation and Shocks', IEEE Trans. Power Deliv., pp. 1-1, 2016.

13. P. Cirillo and J. Hüsler, 'Extreme shock models: An alternative perspective', Stat. Probab. Lett., vol. 81, no. 1, pp. 25-30, Jan. 2011.

14. C. J. Lu and W. O. Meeker, 'Using Degradation Measures to Estimate a Time-to-Failure Distribution', Technometrics, vol. 35, no. 2, pp. 161-174, May 1993.

15. Y. Zhang, H. Liao, and Y. Hong, 'Planning accelerated destructive degradation tests with initiation time', in 2015 Annual Reliability and Maintainability Symposium (RAMS), 2015, pp. 1-6.

16. J. Pan, S. W. Xu, W. H. Chen, X. Y. Wang, P. Qian, and Q. W. Hu, 'Accelerated Aging Test and Study of Storage Life Prediction of NBR O-Ring', Adv. Mater. Res., vol. 415-417, pp. 184-190, Dec. 2011.

17. C. Peng, X. H. Gu, and K. Xiao, 'Prediction for ORing Type Sealing Ring's Storage Life Based on Accelerated Ageing Data', Appl. Mech. Mater., vol. 433-435, pp. 2096-2100, Oct. 2013. 